Document downloaded from:

http://hdl.handle.net/10251/92322

This paper must be cited as:

Bogoni, A.; Petropoulos, P.; Rafel, A.; Teixeira, A.; Fernandez-Palacios Gimenez, JP.; Muñoz Muñoz, P.; Killey, R.... (2016). Foreword to the Special Issue on European Conference on Optical Communications(ECOC 2015). Journal of Lightwave Technology. 34(5):1406-1410. doi:10.1109/JLT.2016.2528358

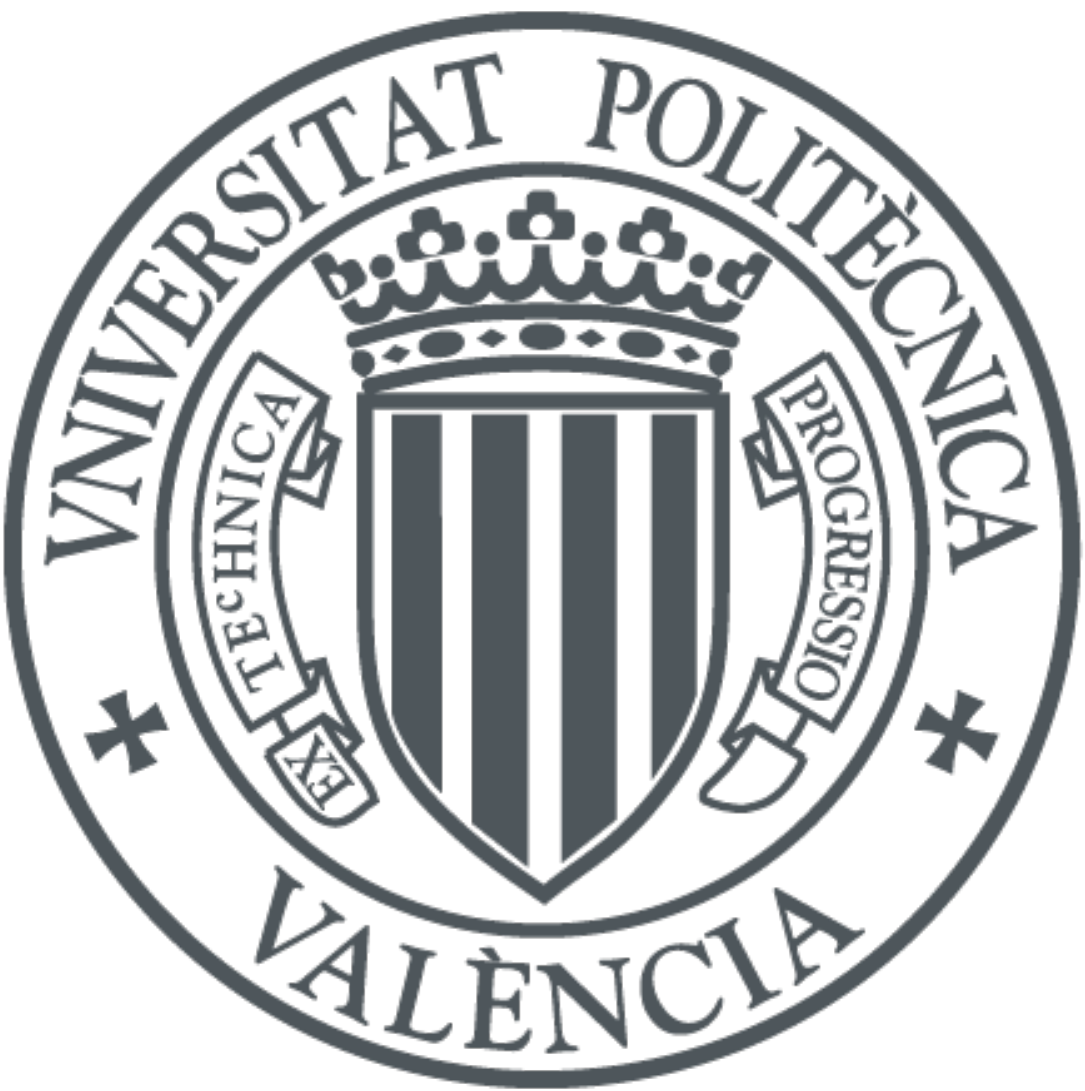

The final publication is available at

http://doi.org/10.1109/JLT.2016.2528358

Copyright Institute of Electrical and Electronics Engineers

Additional Information 


\section{Foreword to the Special Issue on European Conference on Optical Communication (ECOC 2015)}

Every year, and this 41st edition was no exception, ECOC is the leading forum in Europe for keeping up with the top research advances and scientific discoveries in devices, subsystems, systems and networks in the field of optical communications and related photonic technologies. With a global attendance and delegates coming from all over the five continents, ECOC provides a unique opportunity for networking and interaction that nobody interested in the field should miss. ECOC 2015 took place from September $27^{\text {th }}$ to October $1^{\text {st }} 2015$ in Valencia, Spain. As ECOC 2015 organizers, we believe that it is very important to keep the traditions that have contributed to its long-standing success, but at the same we tried to experiment and innovate both in the technical and social programmes. For the first time, ECOC 2015 held a young researcher's meeting and included a Rump Session to debate on the future of optical innovation; "Optical innovation, quo vadis?". ECOC 2015 also held two technical symposia, both of which were unique and special. 2015 was the international Year of Light and ECOC 2015 included a special commemoration symposium, "Light: Enabling the Global Internet Era", at which distinguished keynote speakers addressed how optical technologies have been responsible for the transformation in communications, the evolution of the Internet era as we have experienced it in the last two decades, and why they will play an ever-increasing role in tomorrow's communication networks. A second symposium on one of the hottest topic in the optical community, "Optical Communications and Networks for Datacenters", held in remembrance of our colleague Professor Harm Dorren. It was, focused on discussing the requirements, challenges and solutions for the next-generation datacenters.

The videos of the Plenary speakers (Sir David Payne, Director of the Optoelectronics Research Centre, University of Southampton; Mr. Enrique Blanco, Global CTO of Telefónica, Spain; Dr. Thibaut Kleiner, Head of Unit "Network Technologies" at the European Commission DG CONNECT and Dr. Andreu Vea, Digital Champion for Spain) and the Year of Light symposium speakers (John Harvey, Vice President of the International Commission on Optics The International Year of Light and Light-based Technologies, Emmanuel B. Desurvire, Thales Research \& Technology, Meint K. Smit, COBRA Research Institute, Technical Univ Eindhoven, Neal S. Bergano, TE Subsea Communications, LLC Undersea Fiber Optic Cable Systems and Andrew R. Chraplyvy, Bell Labs) can be found at www.ecoc2015.org. Six successful workshops were held on Sunday afternoon, with a very high participation rate. Well-known industry leaders and internationally recognized academic researchers shared their critical thoughts and wide knowledge on the research needs, requirements, solutions, or market trends on very important topics. In particular, the following workshops were organized:

- Multimode photonics: optical waveguides, components and systems

- How should we design optical communication networks with flexible DSP based transceivers?

- Microwave Photonics

- Optical technologies for the exascale cloud datacenter era

- SDN \& NFV: Real value with new business opportunities or research hype with unmanageable complexity? 
- Multimode photonics: optical waveguides, components and systems

\section{OVERVIEW OF THE SPECIAL ISSUE}

As Guest Editors and Members of the Technical Program Committee of ECOC 2015, it is our pleasure to introduce this special issue of IEEE/OSA JOURNAL OF LIGHTWAVE TECHNOLOGY, as a forum for the authors of the most meaningful contributions of the conference. At ECOC 2015, the papers were categorized into five types: 1) tutorial papers, designed to be instructional lessons made for a broad audience to discover a field, 2) invited oral papers, which provide a synthetic overview of recent results on an important topic, 3) regular oral papers, 4) posters, and 5) postdeadline papers, which report new and significant research in rapidly advancing areas at the earliest possible opportunity. Out of all authors of the $\sim 425$ papers at ECOC 2015, we invited about a quarter of them to write an expanded version of their work for this special issue. We made a first selection according to the following rules. The authors of all tutorial papers were proposed to write an invited tutorial; the authors of all invited oral papers were proposed to write an invited review paper; the authors of the $10 \%$ best-scored regular oral papers were offered the opportunity to write an invited paper; while the authors of all postdeadline papers were proposed to write an invited post-deadline paper. We were delighted to witness that a large majority of the shortlisted authors accepted the invitation.

ECOC2015 continued the new distribution in seven main areas that was offered last year for the first time. The technical content of each one was supervised by a specific subcommittee that, as tradition rules, was composed by world renowned experts and chaired by distinguished scientist. All in all, an impressive Technical Programme Committee composed of 109 members selected a total of $\sim 425$ oral and poster presentations, which include a blend of tutorials, invited and contributed and post deadline papers. The seven areas are:

- (SC1) Fibres, fibre devices and fibre amplifiers

- (SC2) Waveguide and optoelectronic devices

- (SC3) Digital and optical signal processing

- (SC4) Subsystems for optical networking and for datacoms

- (SC5) Point-to-point transmission systems

- (SC6) Core, metro and data center networks

- (SC7) Access, local area and home networks

The special issue on ECOC 2015 spreads over four consecutive issues of JOURNAL OF LIGHTWAVE TECHNOLOGY. The invited tutorials and invited review papers have been allocated in the first issue. The postdeadline invited papers and invited papers are split in the second, third, and fourth issues, according to the topical area where they belonged, when they were accepted at ECOC 2015, namely. 
Following the trend of last years, spatial division multiplexing, datacenter communications, advanced modulation formats, DSP post-processing in coherent systems, and transport software defined network (SDN) control and orchestration continue to be hot topics, while the programme also includes rising star topics such as flexgrid and superchannels, advanced Silicon and InP integrated optics, multicore fiber technologies, MIMO communications and radio over fiber technologies for emerging 5G, access and in-home networks and network function virtualization (NFV).

SC1 highlights include advances in fiber and amplifier technologies for spatial division multiplexing, the development and nonlinear applications of microstructured and few-mode fibers, as well as precise engineering and characterisation of the properties of specialty optical fibers.

Regarding opto-electronic components, highlighted papers deal with modulators and lasers for different formats, in InP or hybridly integrated with Silicon".

Among the most significant papers of $\mathrm{SC}$, the readers will hear about modeling, design, and implementation of digital and/or optical techniques, for signal processing for long-haul, metro or access networks. It also includes digital signal processing algorithms for transmitters and coherent receivers, error correction coding optical regeneration and any other schemes for impairment mitigation.

Regarding the SC4, the reader will find several of the latest advances in real time signal processing targeting improving modulation efficiency at high data rates. Recurring to monolithic photonic integration, advanced techniques for signal, band and sub-band switching and label switching the works challenge the advances in optical and data center networks.

The highlights from the papers presented in the SC5 included advances in fiber nonlinearity mitigation, through coding and constellation optimization, symbol-rate optimization, mid-span spectral inversion and advanced digital nonlinearity compensation such as filtered digital backpropagation. The reader will find results of modelling nonlinear signal-noise interaction with the enhanced Gaussian noise model. Also significant advances in space division multiplexing and novel research on undersea transmission can find out.

The papers in SC6 address several hot topics in the field of transport and data center networks. In particular, SDN/NFV architectures and algorithms applied to multi-tenant, multi-domain and multi-vendor elastic networks, sliceable and virtualizable BVT for rate/distance adaptation, mobile backhaul networks, or hierarchical OAM monitoring have been of major interest. Another major research topic is data center networks, including studies on FPGA-based programmable interfaces, scalable monitoring and optimization, virtual machine migration or OCS/OPS multicast/unicast switch-over.

Finally, SC7 papers cover a number of very important topics on the application and innovation aspects. Of major interest are coherent detection application to PON systems, mobile fronthaul and backhaul applications, and fixed-wireless converged access systems. No less interesting are the investigations on attaining high line bit rates suitable for access systems, plastic fiber connectors, and multi-mode PONs. Finally, NG-PON2 also attracts attention with a 
paper proposing an optical amplification based high power budget, and a tutorial on its Transmission convergence Layer.

The readers can find out about a new momentum for research on optical packet switching, including a considerable effort into operation, design, and fabrication of all optical large port count switches.

The readers can also learn about the latest milestones along the endless path toward greater capacities, in particular, in the list of invited postdeadline papers. Along this path, some authors discuss new advances in digital mitigation techniques against nonlinear distortions in fibers.

The readers can figure out how the aspiration for networks function virtualization (NFV) and automation could reshape the transport network. This special issue also confirms that optical technologies can alleviate the load of electronics, for example, by performing dynamic allocation of resources and switching.

Finally, the special issue explores next generation solutions of passive optical network (PON) technologies. The next generation PON2 topic concerns several papers and considers the way to deliver very high bit rate inside the home network by record free-space optic links.

In summary, this special issue brings to the readers of JOURNAL OF LIGHTWAVE TECHNOLOGY a quite comprehensive overview of the most significant activities on optical communication in the second half of 2015. As such, we hope that it helps the researchers from outside our community to discover the field, and that it becomes a reference document on our shelves.

\section{ACKNOWLEDGMENT}

The Guest Editors would like to thank the editorial staff for their support. We are also grateful to all the reviewers who did a great job, despite the strong timing constraints, particularly to the Members of Technical Program Committee of ECOC 2015, who were heavily involved in the reviews. 